\title{
Síndrome de Guillain-Barré em associação temporal com a vacina influenza A
}

\author{
Guillain-Barré syndrome in temporal association with influenza A vaccine
}

Maria Aparecida G. Ferrarini', Mônica Ayres A. Scattolin², Marcelo Masruha Rodrigues ${ }^{3}$, Maura Helena F. Resende ${ }^{4}$, Isabel Cristina L. dos Santos ${ }^{5}$, Antônio Vladir lazzetti ${ }^{6}$

\section{RESUMO}

Objetivo: Descrever um caso de síndrome de GuillainBarré em associação temporal com a vacina influenza A (H1N1) 2009.

Descrição do caso: Menino de quatro anos com queixa inicial de dor em coxa direita e perda de força muscular ascendente 15 dias após a segunda dose da vacina influenza A (H1N1) 2009. Ao exame neurológico apresentava tetraparesia e arreflexia, com predomínio em membros inferiores. A eletroneuromiografia evidenciou redução da velocidade e bloqueio de condução neuronal, com discreta perda axonal secundária. Foi tratado com imunoglobulina por via intravenosa, atingiu platô no quarto dia de evolução da doença e, depois, houve melhora progressiva da força muscular.

Comentários: Com o emprego em larga escala da vacina influenza A (H1N1) 2009 em nosso meio e os dados preliminares do sistema de vigilância norte-americano mostrando associação temporal significante com a síndrome de GuillainBarré, recomenda-se a descrição dos casos suspeitos dessa associação. A vacina continua sendo o método mais efetivo para prevenir doença grave e morte por influenza.

Palavras-chave: vacinas contra influenza/efeitos adversos; síndrome de Guillain-Barré; influenza/prevenção \& controle.

\section{ABSTRACT}

Objective: To report a case of Guillain-Barré syndrome following influenza A (H1N1) 2009 vaccine.

Case description: A four-year-old boy presented right thigh pain and ascending muscular weakness 15 days after the second dose of influenza A (H1N1) 2009 vaccine. The neurological examination revealed tetraparesis and areflexia. Electroneuromyography showed lower velocity and conduction blockage with small secondary axonal loss. Treated with intravenous immunoglobulin, the patient reached a plateau in the $4^{\text {th }}$ day, followed by progressive muscular strength improvement.

Comments: The employment of large-scale influenza A (H1N1) 2009 vaccination and the preliminary reports from the American Surveillance Program suggest a significant association between Guillain-Barré syndrome and influenza A H1N1 2009 vaccination. All suspected cases of this association should be published for further evaluation. Vaccination remains the most effective method to prevent serious illness and death related to influenza.

Key-words: influenza vaccines/adverse effects; GuillainBarre syndrome; influenza/prevention \& control.
Instituição: Universidade Federal de São Paulo (Unifesp), São Paulo, SP, Brasil

'Mestre em Pediatria pela Unifesp; Médica Assistente da Disciplina de Infectologia Pediátrica do Departamento de Pediatria da Unifesp, São Paulo, SP, Brasil

${ }^{2}$ Especialista em Neuropediatria pela Unifesp, São Paulo, SP, Brasil

${ }^{3}$ Doutor em Ciências pela Unifesp; Professor Adjunto do Departamento de Neurologia e Neurocirurgia da Unifesp, São Paulo, SP, Brasil

${ }^{4}$ Residente em Pediatria da Unifesp, São Paulo, SP, Brasil

${ }^{5}$ Residente em Infectologia Pediátrica da Unifesp, São Paulo, SP, Brasil ${ }^{6}$ Mestre em Pediatria pela Unifesp; Professor Adjunto da Disciplina de Infectologia Pediátrica do Departamento de Pediatria da Unifesp, São Paulo, SP, Brasil
Endereço para correspondência:

Maria Aparecida G. Ferrarini

Rua Loefgreen, 1.998 - Vila Clementino

CEP 04040-003 - São Paulo/SP

E- mail: dipe@epm.br

Conflito de interesse: nada a declarar

Recebido em: 15/10/2010

Aprovado em: 2/3/2011 


\section{Introdução}

A síndrome de Guillain-Barré (SGB) é caracterizada por uma polineuropatia inflamatória desmielinizante aguda, que compromete sobretudo os nervos periféricos e leva a graus variados de fraqueza motora progressiva e ascendente, podendo atingir os membros superiores e a face. É acompanhada de anormalidades sensoriais e disfunções autonômicas, com consequente perda dos reflexos tendinosos profundos ${ }^{(1-3)}$.

A SGB é a causa mais frequente de paralisia flácida aguda e subaguda desde a erradicação da poliomielite. A incidência no mundo é de 0,6 a quatro casos por 100.000 habitantes e aumenta com a idade, sendo os homens 1,5 vezes mais afetados $^{(1)}$. Na América Latina e no Caribe, a incidência média é de 0,82 casos $/ 100.000$ crianças menores de 15 anos

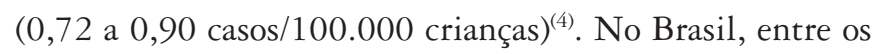
anos de 1990 e 1996, a incidência anual foi de 0,39 a 0,63 casos/100.000 habitantes, em menores de 15 anos $^{(5)}$. Em um estudo na cidade de São Paulo, entre 1995 e 2002, a incidência anual foi de 0,6 casos por 100.000 habitantes $^{(2)}$.

A etiologia é desconhecida, mas, em aproximadamente dois terços dos casos, há relato de infecção respiratória ou gastrintestinal nas seis semanas que precederam o quadro e, raramente, pode seguir-se à vacinação ${ }^{(1,6)}$. As considerações sobre o risco de desenvolver a SGB após a vacinação da influenza existem desde a associação do primeiro caso descrito em 1976, durante a campanha nacional de imunização contra a vacina influenza A/New Jersey suína, nos Estados Unidos. Nessa ocasião houve um aumento do risco em aproximadamente seis vezes de desenvolver SGB até 42 dias após a vacina (acréscimo de dez casos/1 milhão de vacinados), sendo indicada a interrupção da vacinação devido ao contexto de baixa transmissão do vírus da influenza ${ }^{(3,6)}$.

A monitoração da segurança da vacina (monovalente e fragmentada) influenza A (H1N1) empregada em 2009 é realizada por uma rede de sistemas de vigilância nos Estados Unidos, e resultados preliminares do Centers for Disease Control and Prevention (CDC) mostram uma associação significante entre a vacina da influenza $A(\mathrm{H} 1 \mathrm{~N} 1)$ 2009 e a SGB, com aumento do risco em 0,8 casos/1 milhão de vacinados ${ }^{(6)}$. Se esses dados forem confirmados no final do período de vigilância, o risco pode ser comparável ao previamente descrito para a vacina da influenza sazonal (aproximadamente um caso/1 milhão de vacinados) e muito menor que o risco de SGB observada na campanha de 1976. Outros sistemas de vigilância não detectaram qualquer associação entre SGB e vacina influenza A (H1N1) 2009 monovalente ${ }^{(6,7)}$.

Nesse contexto, o objetivo desse relato é descrever um caso de SGB em associação temporal com a vacina influenza A (H1N1) 2009, com a aprovação do Comitê de Ética em Pesquisa da Universidade Federal de São Paulo (Unifesp).

\section{Descrição do caso}

Pré-escolar com quatro anos de idade, masculino, branco apresentou dor e parestesia em coxa direita dois dias antes da admissão hospitalar, que progrediram para ambos os membros inferiores, acompanhadas de perda de força, atingindo um platô no quarto dia de evolução ao comprometer membros superiores. Apresentava histórico de ataxia cerebelar pós-infecciosa secundária a quadro de varicela aos três anos de idade. A vacinação básica estava completa, tendo recebido duas doses da vacina influenza A (H1N1) 2009 (Sanofi- Pasteur- lote UP 137AA) com intervalo de 40 dias entre as doses, sendo a última há 15 dias do início da queixa.

Ao exame físico geral encontrava-se em bom estado, sem anormalidades a não ser os sinais neurológicos, com peso e estatura adequados para idade. O exame neurológico revelava arreflexia e tetraparesia com predomínio em membros inferiores, estando os graus de força descritos na Tabela 1. Não houve perda de força da musculatura bulbar.

Tabela 1 - Graus de força em paciente com dois dias de evolução da síndrome de Guillain-Barré

\begin{tabular}{|c|c|c|c|c|c|c|c|c|}
\hline \multicolumn{9}{|c|}{ Força muscular } \\
\hline $\begin{array}{l}\text { Membros } \\
\text { Superiores }\end{array}$ & Flexão & Extensão & Abdução & Adução & Pronação & Supinação & Preensão & Oponência \\
\hline & 2 & 3 & 3 & 3 & 3 & 3 & 3 & 3 \\
\hline $\begin{array}{l}\text { Membros } \\
\text { inferiores }\end{array}$ & Flexão & Extensão & Abdução & Adução & Dorsiflexão & $\begin{array}{l}\text { Flexão- } \\
\text { Plantar }\end{array}$ & Eversão & Inversão \\
\hline & 2 & 2 & 1 & 1 & 1 & 1 & 1 & 1 \\
\hline
\end{tabular}

0: sem contração; 1: contração muscular palpável ou visível sem deslocamento; 2 : contração muscular ativa com deslocamento sem vencer a gravidade; 3: contração muscular ativa com deslocamento e vence a gravidade; 4: contração muscular ativa com deslocamento e vence resistência moderada; 5: normal. 
A análise do líquor, no terceiro dia de evolução, não apresentou dissociação proteino-citológica (celularidade: 3,3; glicose: $60 \mathrm{mg} / \mathrm{dL}$, proteínas: $30,4 \mathrm{mg} / \mathrm{dL}$ [referência: 25 a $45 \mathrm{mg} / \mathrm{dL}]$ ). A eletroneuromiografia demonstrou prolongamento das ondas $\mathrm{F}$, redução da velocidade de condução neuronal e bloqueio de condução, com discreta perda axonal secundária. Apresentou reações sorológicas negativas para quadros agudos de mononucleose infecciosa, toxoplasmose, citomegalovírus e vírus da imunodeficiência humana, além de pesquisa negativa para Campylobacter spp nas fezes.

Recebeu imunoglobulina humana intravenosa na dose total de $2 \mathrm{~g} / \mathrm{kg}$ em quatro dias, sem intercorrências. O paciente apresentou melhora progressiva, rápida, com retorno da deambulação sem apoio no sétimo dia, e remissão completa dos déficits neurológicos em nove dias.

\section{Discussão}

A SGB é uma doença imunomediada, descrita pela primeira vez em 1916 pelos neurologistas franceses Guillain, Barré e Strohl ${ }^{(8)}$, resultando da produção de autoanticorpos com reação cruzada com epítopos dos nervos e raízes nervosas, o que leva à desmielinização e ao dano axonal ${ }^{(9,10)}$.

O desafio antigênico, seja por uma infecção prévia ou imunização, estimula uma reação humoral e/ou celular antígenoespecífica que pode, em teoria, desencadear a SGB. Uma explicação possível é o mecanismo de mimetismo molecular, no qual patógeno e hospedeiro compartilham sequências de aminoácidos idênticas ou homólogas, e os receptores de célula $\mathrm{B}$ e $\mathrm{T}$ do hospedeiro reconhecem esses peptídeos e desencadeiam a doença imunomediada ${ }^{(1,11)}$.

A doença, em geral, é precedida por infecções respiratórias e gastrintestinais, com destaque para as infecções pelo Campylobacter jejuni. Outros agentes descritos em associação temporal são o vírus Epstein-Barr, citomegalovírus, Haemophilus influenzae, Mycoplasma pneumonia ${ }^{(1)}$ e Borrelia burgdorferi ${ }^{(12,13)}$.

As considerações sobre a associação entre as vacinas influenza e SGB iniciaram-se após os eventos descritos com a vacina influenza $\mathrm{A} / \mathrm{New}$ Jersey suína, nos Estados Unidos, em $1976^{(1)}$. Os estudos subsequentes que investigaram a associação entre SGB e a vacina da influenza sazonal relataram baixo risco relativo, sem significância estatística ${ }^{(1,9)}$. As vacinas da raiva, pólio oral, difteria, toxoide tetânico, hepatite $\mathrm{B}$, meningocócica conjugada foram também associadas temporalmente à SGB, porém não foi estabelecida qualquer conclusão de causalidade ${ }^{(1,14)}$.
Dados do Vaccine Adverse Event Reporting System (VAERS) do CDC norte-americano demonstraram que, entre 1990 e 2003, a despeito do aumento de notificações de diversos eventos adversos relacionados às vacinas influenza utilizadas nesse período, houve decréscimo nas notificações de SGB, especialmente após o inverno de 1996-1997(9,15). O estudo mencionado sugeriu a possibilidade de uma associação causal da vacina com a SGB devido a dois achados: o primeiro foi a pequena porcentagem de pessoas que tiveram doença precedendo a SGB nas quatro semanas anteriores à vacinação e o segundo fato foi a presença de um intervalo longo (13 dias) para o início do quadro versus o intervalo curto (um dia) para os outros eventos adversos que não a SGB ${ }^{(14,15)}$.

Estudo recente, com a UK General Practice Research Database no período de 1990 a 2005, não encontrou aumento do risco de SGB no período de 90 dias após a vacina da influenza sazonal $^{(7)}$. Porém, comprovou-se um risco aumentado de SGB após a doença influenza like, principalmente no mês subsequente à infecção ${ }^{(7)}$.

O diagnóstico da SGB usualmente se baseia na apresentação clínica. Os exames subsidiários são úteis para excluir outros diagnósticos e para fornecer subsídios a respeito do prognóstico e do estado funcional. O liquor da maioria dos pacientes apresenta hiperproteinorraquia e celularidade baixa ou normal (dissociação proteíno-citológica), podendo estar normal em até uma a duas semanas após o início da queixa ${ }^{(16)}$.

$\mathrm{O}$ caso descrito apresentou uma relação temporal entre a aplicação da segunda dose da vacina da influenza pandêmica 2009 e a SGB. O intervalo de tempo estabelecido para esse tipo de associação na literatura é de até seis semanas ${ }^{(17)}$. Embora o quadro anterior de ataxia cerebelar pós-infecciosa levante a hipótese de que a estimulação imunológica desse paciente possa predispô-lo a doenças imunomediadas, é importante destacar apenas a existência comprovada de uma associação temporal e não causal entre a aplicação da vacina descrita e a SGB.

A publicação deste caso de SGB com associação temporal provável com a vacina da influenza tem o sentido de informar a comunidade científica sobre essa possibilidade e, talvez, somada a outros relatos, aponte a necessidade de pesquisas futuras sobre essa potencial associação. $\mathrm{Na}$ literatura, o primeiro caso pediátrico de SGB, em associação temporal com a vacina da influenza pandêmica 2009, foi descrito no Canadá(18).

É importante reforçar que a SGB é um evento extremamente raro e os benefícios da vacina da influenza são indiscutíveis. A vacina permanece o método mais efetivo para prevenir a doença ${ }^{(19)}$. 


\section{Referências bibliográficas}

1. Pithadia $A B$, Kakadia N. Guillain-Barré syndrome (GBS). Pharmacol Rep 2010;62:220-32.

2. Rocha MS, Brucki SM, Carvalho AA, Lima UW. Epidemiologic features of Guillain-Barré syndrome in São Paulo, Brazil. Arq Neuropsiquiatr 2004;62:33-7.

3. Van Doorn PA, Ruts L, Jacobs BC. Clinical features, pathogenesis, and treatment of Guillain-Barre syndrome. Lancet Neurol 2008;7:939-50.

4. Landaverde JM, Danovaro-Holliday MC, Trumbo SP, Pacis-Tirso CL, RuizMatus $C$. Guillain-Barré syndrome in children aged $<15$ years in Latin America and the Caribbean: baseline rates in the context of the influenza A (H1N1) pandemic. J Infect Dis 2010;201:746-50.

5. Dias-Tosta E, Kückelhaus CS. Guillain Barré syndrome in a population less than 15 years old in Brazil. Arq Neuropsiquiatr 2002;60:367-73.

6. Centers for Disease Control and Prevention (CDC). Preliminary results: surveillance for Guillain-Barre syndrome after receipt of influenza A (H1N1) 2009 monovalent vaccine - United States, 2009-2010. MMWR Morb Mortal Wkly Rep 2010;59:657-61.

7. Stowe J, Andrews N, Wise L, Miller E. Investigation of the temporal association of Guillain-Barre syndrome with influenza vaccine and influenza-like illness using the United Kingdom General Practice Research Database. Am J Epidemiol 2009;169:382-8.

8. Asbury AK. Guillain-Barre syndrome: historical aspects. Ann Neurol 1990;27 (Suppl):S2-6.

9. Evans D, Cauchemez S, Hayden Frederick G. "Prepandemic" immunization for novel influenza viruses, "swine flu" vaccine, Guillain-Barré syndrome, and the detection of rare severe adverse events. J Infect Dis 2009;200:321-8.

10. Israeli E, Agmon-Levin N, Blank M, Chapman J, Shoenfeld Y. Guillain-Barre syndrome-a classical autoimmune disease triggered by infection or vaccination.
Clin Rev Allergy Immunol 2010 Oct 5. [Epub ahead of print].

11. Kalume F, Lee SM, Morcos Y, Callaway JC, Levin MC. Molecular mimicry:crossreactive antibodies from patients with immune-mediated neurologic disease inhibit neuronal firing. J Neurosci Res 2004;77:82-9.

12. Tam CC, O'Brien SJ, Petersen I, Islam A, Hayward A, Rodrigues LC. GuillainBarre syndrome and preceding infection with campylobacter, influenza and Epstein-Barr virus in the general practice research database. PLoS One 2007:2:e344.

13. Shapiro EE. Guillain-Barre syndrome in a child with serologic evidence of Borrelia burgdorferi infection. Pediatr Infect Dis J 1998;17:264-5.

14. Haber P, Sejvar J, Mikaeloff $Y$, DeStefano F. Vaccines and Guillain-Barre syndrome. Drug Saf 2009;32:309-23.

15. Zhou W, Pool V, Iskander JK, English-Bullard R, Ball R, Wise RP et al. Surveillance for safety after immunization: Vaccine Adverse Event Reporting System (VAERS)--United States, 1991-2001. MMWR Surveill Summ 2003;52:1-24.

16. Brettschneider J, Petzold A, Süssmuth S, Tumani H. Cerebrospinal fluid biomarkers in Guillain-Barre syndrome--where do we stand? J Neurol 2009;256:3-12.

17. Sejvar JJ, Kohl KS, Gidudu J, Amato A, Bakshi N, Baxter R et al. GuillainBarre syndrome and Fisher syndrome: case definitions and guidelines for collection, analysis, and presentation of immunization safety data. Vaccine 2011;29:599-612.

18. Tremblay ME, Closon A, D'Anjou G, Bussieres JF. Guillain-Barre Syndrome Following H1N1 Immunization in a Pediatric Patient. Ann Pharmacother 2010;44:1330-3.

19. Lehmann HC, Hartung HP, Kieseier BC, Hughes RA. Guillain-Barre syndrome after exposure to influenza virus. Lancet Infect Dis 2010;10:643-51. 